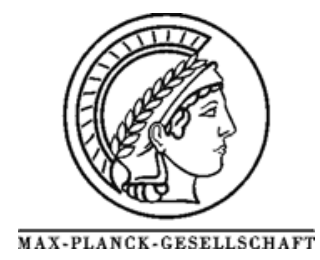

\title{
How important is the (001) plane of M1 for selective oxidation of propane to acrylic acid?
}

\author{
A. Celaya Sanfiz ${ }^{\mathrm{a}}$, T.W. Hansen ${ }^{\mathrm{a}}$, A. Sakthivel ${ }^{\mathrm{a}}$, A. Trunschke ${ }^{\mathrm{a} *}$, R. Schlögl ${ }^{\mathrm{a}}$ \\ A. Knoester ${ }^{\mathrm{b}}$, H.H. Brongersma ${ }^{\mathrm{b}}$ \\ M.H. Looi ${ }^{\mathrm{c}}$, S.B.A. Hamid ${ }^{\mathrm{c}}$ \\ ${ }^{a}$ Fritz Haber Institute of the Max Planck Society, Department of Inorganic Chemistry, \\ Faradayweg 4-6, D-14195 Berlin, Germany \\ ${ }^{b}$ Calipso B.V., P.O. Box 513, 5600 MB Eindhoven, The Netherlands

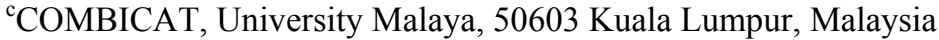

${ }^{*}$ Corresponding author: e-mail trunschke@,fhi-berlin.mpg.de,

Received Received 7 March 2008; Revised 22 May 2008; Accepted 23 May 2008; Available online 3 July 2008.

\begin{abstract}
The role of the (001) crystallographic plane of the M1 phase of MoVTeNb mixed oxide catalysts in selective oxidation of propane to acrylic acid has been addressed by investigating a phase-pure M1 material preferentially exposing this surface. A model catalyst has been prepared by complete silylation of $\mathrm{M} 1$ followed by breakage of the $\mathrm{SiO}_{2}$ covered needles. Using this approach, the reactivity of the M1 (001) surface has been investigated by combining a micro-reactor study of propane oxidation with High-Sensitivity Low Energy Ion Scattering (HS-LEIS). Scanning electron microscopy (SEM) and transmission electron microscopy (TEM) have been used to study shape and microstructure of the model system and to verify the surface exposure of the model catalyst. The specific formation rate of acrylic acid on the model catalyst is similar to that on the phase-pure M1 reference material indicating that the (001) plane of the M1 crystal structure does not possess enhanced catalytic properties compared to the lateral surface of M1 needles in propane oxidation.
\end{abstract}

Keywords: MoVTeNb Oxide Catalyst; M1; Propane Oxidation; Propane Ammoxidation; Low-Energy lon Scattering; LEIS

\section{Introduction}

Selective oxidation of light alkanes requires multifunctional catalysts capable of simultaneous activation of $\mathrm{C}-\mathrm{H}$ bonds, insertion of oxygen atoms, and prevention of $\mathrm{CO}_{\mathrm{x}}$ formation by further oxidation of intermediates or desired reaction products, which are usually much more reactive than the inactive reactant. The necessary functional diversity is implemented in chemically and structurally complex $\mathrm{MoVTeNbO}_{\mathrm{x}}$ catalysts, which show high activities and selectivities in partial (amm)oxidation of propane to acrylic acid and acrylonitrile, respectively $[1,2]$. Usu- ally, these catalysts consist of phase mixtures composed of the so-called "M1" and "M2" phases [3], as well as minor amounts of phases, like $\mathrm{Mo}_{5} \mathrm{O}_{14}$-type structures or binary MoV and MoTe oxides. Propane activation and high selectivities towards acrylic acid and acrylonitrile are generally attributed to the presence of the orthorhombic M1 phase [46]. The M1 structure (ICSD 55097) consists of cornersharing $\mathrm{MO}_{6}$ octahedrons, $(\mathrm{M}=\mathrm{Mo}, \mathrm{V})$, which are assembled in the (001) plane forming characteristic hexagonal and heptagonal rings hosting Te-O units. Niobium is preferentially located in pentagonal bipyramidal environment [7-9]. However, due to a certain chemical flexibility of the 


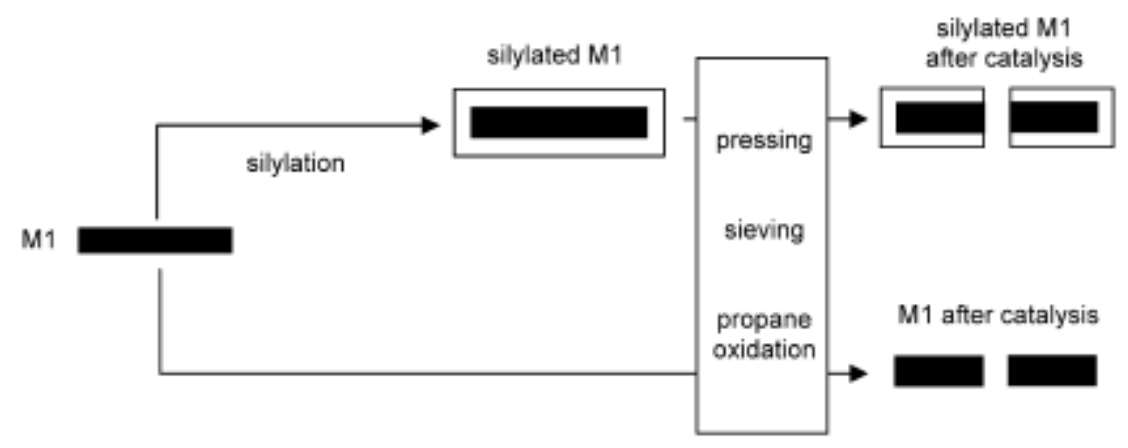

Scheme 1: Silylation and mechanical treatment of phase-pure M1

phase, octahedral positions may also be occupied by $\mathrm{Nb}$ in $\mathrm{M} 1$ with higher $\mathrm{Nb}$ content [10]. A bronze like channel structure is established by stacking layers of the polyhedrons in the [001] direction resulting in a needle-like crystal morphology in which the $\{001\}$ planes are arranged perpendicular to the length axis of the needle. It has been suggested that terminating (001) planes of the M1 phase contain the active and selective surface sites for partial oxidation reactions [11-14]. This claim has stimulated research on the structure and properties of the M1 surface by Low Energy Ion Scattering (LEIS) $[15,16]$. One of the unique features of LEIS analysis is that it gives the atomic composition of the outermost atoms of a surface. These outer atoms are precisely the atoms that largely control the catalytic properties of the solid. It has been shown before [17] that in cases where conventional surface analytic techniques, such as XPS, do not show correlation with the catalytic activity, the extreme surface sensitivity of LEIS gives a direct relationship between composition and catalysis. In a recent review [18], the underlying principles and quantification of LEIS are described. The general absence of matrix effects enables the use of simple reference samples for the quantification of the surface composition.

The present study addresses the origin of the positive effect of grinding on the catalytic activity of MoVTeNb mixed oxides in the selective oxidation of propane to acrylic acid. Due to the needle-shape of the particles, the surface fraction of (001) planes comprises only a minor fraction of the total surface area. Thus, increased activity after grinding of M1 needles has been attributed to the generation of additional (001) surface area [11, 12]. Even though an increased specific surface area is observed [12], the downside of the grinding process is the well known impact of mechanical treatment on the nature and concentration of defects on the overall surface of transition metal oxides [19].

Therefore, a different strategy illustrated in Scheme 1 has been pursued. A batch of crystalline, phase-pure M1 prepared by hydrothermal synthesis was divided into two parts. One part was fully coated with silica. The complete coverage of the mixed metal oxide surface by $\mathrm{SiO}_{2}$ was verified by HS-LEIS. Both, the coated material and the non-coated reference M1, were pressed into pellets, crushed and sieved in order to prepare sieve fractions, which were then loaded into a micro-reactor and studied in the partial oxidation of propane to acrylic acid. The preparation of the sieve fraction represents a gentle mechanical treatment that generates new M1 surface, which has been quantified by LEIS for the silica-coated catalyst [18] and by nitrogen adsorption for the reference material. Special attention has been paid to a comprehensive microstructural characterization of the model catalysts before and after the propane oxidation. Scanning electron microscopy (SEM) and transmission electron microscopy (TEM) revealed that complete coverage of M1 followed by breakage of the $\mathrm{SiO}_{2}$-covered needles generates a model catalyst that predominantly exposes (001) planes (Scheme 1). In addition, LEIS was applied to determine the chemical composition of the newly formed M1 surface of the coated catalyst in comparison to the composition of the total surface of the M1 reference. Based on the results of the micro-reactor and LEIS study, the relevance of particular surface terminations will be discussed in view of the catalytic properties of phase-pure M1 catalysts in partial oxidation of propane to acrylic acid.

\section{Experimental}

\subsection{Preparation of $M 1$}

A phase-pure M1 catalyst with nominal atomic ratio $\mathrm{Mo} / \mathrm{V} / \mathrm{Te} / \mathrm{Nb}=1 / 0.3 / 0.23 / 0.13$ has been prepared starting from $18.71 \mathrm{~g} \mathrm{MoO}_{3}$ (Fluka) suspended in $300 \mathrm{ml}$ bidistilled water. After adding $19.90 \mathrm{~g}$ oxalic acid at $363 \mathrm{~K}$ a light yellowish solution has been obtained. The mixture was stirred for 25 minutes and cooled down to $313 \mathrm{~K}$. Afterwards, $6.86 \mathrm{~g}$ of telluric acid (Sigma-Aldrich) dissolved in $30 \mathrm{ml}$ bidistilled water at $313 \mathrm{~K}$ was added to the molybdenum oxalate solution. A vanadium solution was synthesized by carefully mixing $7.05 \mathrm{~g}$ oxalic acid and $3.55 \mathrm{~g} \mathrm{~V}_{2} \mathrm{O}_{5}$ (Riedel-de-Haën) in $30 \mathrm{ml}$ bidistilled water at $338 \mathrm{~K}$. The resulting blue solution was cooled down to $313 \mathrm{~K}$ and added to the binary MoTe solution. Finally, a solution of $7.35 \mathrm{~g}$ ammonium niobium oxalate (Sigma-Aldrich) in $30 \mathrm{ml} \mathrm{bi-}$ distilled water was added at $313 \mathrm{~K}$ to the previous mixture. 
Table 1: Results of particle shape analysis based on SEM images and BET surface areas measured by nitrogen adsorption

\begin{tabular}{|c|c|c|c|c|c|c|c|c|}
\hline Catalyst & $\begin{array}{l}\text { Number } \\
\text { of parti- } \\
\text { cles }\end{array}$ & $\begin{array}{l}\mathbf{I}_{\text {mean }} \\
{[\mathrm{nm}]}\end{array}$ & $\begin{array}{l}\mathbf{d}_{\text {mean }} \\
{[\mathrm{nm}]}\end{array}$ & $d / l$ & $\begin{array}{l}\text { Calculated }^{3} \\
\text { total surface } \\
\text { area of one } \\
\text { needle [nm }{ }^{2} \text { ] }\end{array}$ & $\begin{array}{c}\text { Calculated }^{3} \\
\text { surface area of } \\
\text { the }(001) \text { plane } \\
\text { of one needle } \\
{\left[\mathrm{nm}^{2}\right]}\end{array}$ & $\begin{array}{c}\text { Calculated }^{3} \\
\text { specific sur- } \\
\text { face area } \\
{\left[\mathrm{m}^{2} / \mathrm{g}\right]}\end{array}$ & $\begin{array}{c}\text { BET surface } \\
\text { area } \\
{\left[\mathrm{m}^{2} / \mathrm{g}\right]}\end{array}$ \\
\hline M1 $^{1}$ & 379 & $336 \pm 189$ & $184 \pm 99$ & 0.56 & $2.4710^{5}$ & $0.5310^{5}$ & 6.3 & 6.7 \\
\hline M1 $^{2}$ & 241 & $277 \pm 119$ & $164 \pm 85$ & 0.59 & $1.8510^{5}$ & $0.4210^{5}$ & 7.2 & 7.6 \\
\hline $\begin{array}{l}\text { silyl. } \\
\text { M12 }^{2}\end{array}$ & 298 & $275 \pm 114$ & $167 \pm 71$ & 0.61 & $1.8810^{5}$ & $0.4410^{5}$ & 7.1 & n. d. \\
\hline
\end{tabular}

${ }^{1} \mathrm{M} 1$ as synthesized

2 catalyst after propane oxidation

${ }^{3}$ calculation under consideration of mean diameter, mean length and under assumption of cylindrical geometry with circular basal plane

The clear quaternary solution was stirred for $10 \mathrm{~min}$ and spray-dried in a Büchi B-191 spray-dryer at an inlet temperature of $423 \mathrm{~K}$. The delivery rate of the pump and the aspirator were tuned to an outlet temperature of $383 \mathrm{~K}$.

The spray-dried material was calcined in flowing air at $548 \mathrm{~K}$ (heating rate $5 \mathrm{~K} / \mathrm{min}$ ) for one hour. The calcined mixed oxide was then heated for two hours at $773 \mathrm{~K}$ (heating rate $5 \mathrm{~K} / \mathrm{min}$ ) and $20 \mathrm{MPa}$ in the presence of steam. The resulting solid was finally crystallized to $\mathrm{M} 1$ in flowing argon at $873 \mathrm{~K}$ (heating rate $15 \mathrm{~K} / \mathrm{min}$ ) for another two hours (catalyst ID 3030). The atomic ratio of the metals in the final catalyst $\mathrm{Mo} / \mathrm{V} / \mathrm{Te} / \mathrm{Nb}=1 / 0.34 / 0.08 / 0.14$ as determined by EDX is close to the nominal ratio with the exception of the reduced tellurium content due to evaporation of elemental Te during the thermal activation of the catalyst.

\subsection{Silylation of $M 1$}

Silylation of M1 has been performed by addition of $5.8 \mathrm{~g} \mathrm{HSi}(\mathrm{OEt})_{3}$ (Sigma-Aldrich) to a suspension of $0.5 \mathrm{~g}$ phase-pure M1 (catalyst 3030) in $100 \mathrm{ml}$ toluene. The mixture was kept for 16 hours at $384 \mathrm{~K}$ under reflux. Crosslinking of anchored silanol groups has been achieved by treating the resulting solid in a mixture of $90 \mathrm{ml}$ ethanol, $10 \mathrm{ml}$ of bidistilled water and $1 \mathrm{ml}$ concentrated sulphuric acid for four hours under reflux. The two-step procedure has been repeated three times in order to assure complete silylation of the entire M1 surface (catalyst ID 3159).

\subsection{Activity measurements}

For testing of the catalysts in selective oxidation of propane to acrylic acid, $0.5 \mathrm{~g}$ of each material was pelletized ( 8 ton on a round surface $3 \mathrm{~cm}$ in diameter) and sieved $(200-400 \mu \mathrm{m})$. This shape forming procedure is referred to as mechanical treatment. It causes partial disruption of the needles with the consequence that the size distribution of the primary particles in the original M1 is changed and, moreover, this means that catalytic testing of completely silylated M1 was impossible. However, pure silica (Aerosil 300) has been shown to be inactive in propane oxidation under the reaction conditions applied. The sieve fractions of the mechanically treated M1 and silylated M1, respectively, were loaded into quartz reactors with a diameter of $4 \mathrm{~mm}$. The feed was composed of propane/ oxygen/ nitrogen / steam in a molar ratio of $0.85 / 1.9 / 15.2 / 12$. The reaction has been performed at atmospheric pressure, a GHSV of $4800 \mathrm{~h}^{-1}$, and a reaction temperature of $673 \mathrm{~K}$. The products were analyzed by gas chromatography. A molecular sieve column and a Porapak column coupled with a thermal conductivity detector were used for the analysis of $\mathrm{O}_{2}, \mathrm{~N}_{2}, \mathrm{CO}, \mathrm{CO}_{2}$, and hydrocarbons (C1-C3). The oxygenated products were analyzed applying a HP-FFAP column coupled with a flame ionization detector. The detection limit of the individual products depends on the detector used. It is generally greater than 0.2 vol- $\%$. A mass balance of $100+/-10 \%$ was calculated.

\subsection{X-ray diffraction}

The phase-purity of the materials was verified by $\mathrm{x}$ ray diffraction (XRD). The measurements were performed with a STOE STADI-P transmission diffractometer equipped with a focusing primary $\mathrm{Ge}$ (111) monochromator and a position sensitive detector, using $\mathrm{Cu}-\mathrm{K} \alpha_{1}$ radiation. For data analysis, the program Topas (v.2.1, Bruker AXS) was used to fit the diffraction pattern of the activated materials.

\subsection{Electron microscopy}

The microstructure of the catalysts was investigated by transmission electron microscopy, carried out in a Philips CM 200 FEG transmission electron microscope operated at $200 \mathrm{kV}$. The microscope is equipped with a Gatan CCD camera for image acquisition and an EDAX Genesis EDX system. Morphology studies and shape analysis were performed applying scanning electron microscopy. A Hitachi S-5200 with a PGT Spirit EDX system and a Hitachi S4800 with an EDAX Genesis EDX detector were used. EDX studies in the SEMs were carried out with an accele 


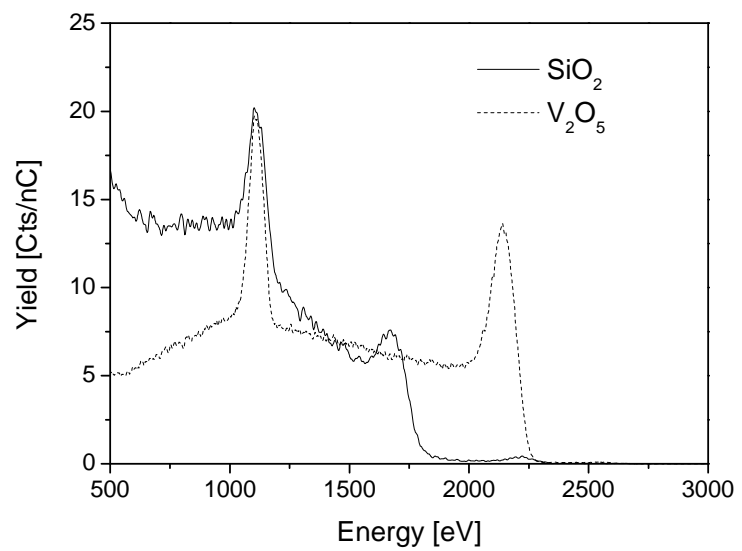

Fig. 1: $3 \mathrm{keV} \mathrm{He}{ }^{+}$scattering. References $\mathrm{SiO}_{2}$ and $\mathrm{V}_{2} \mathrm{O}_{5}$

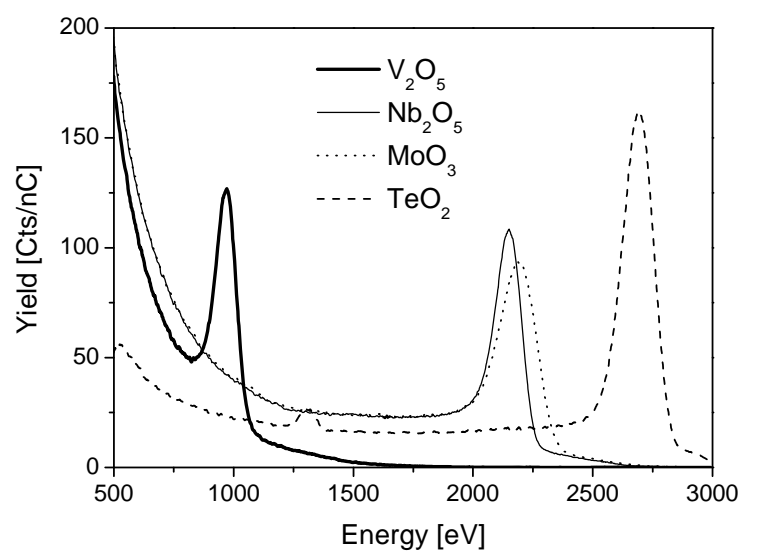

Fig.2: $5 \mathrm{keV} \mathrm{Ne}+$ scattering. references $\mathrm{V}_{2} \mathrm{O}_{5}, \mathrm{Nb}_{2} \mathrm{O}_{5}, \mathrm{MoO}_{3}$ and $\mathrm{TeO}_{2}$

rating voltage of $10 \mathrm{kV}$ while images were acquired at $2 \mathrm{kV}$ to optimize surface resolution. For TEM, the specimens were prepared by dry dispersing the catalyst powder on a standard copper grid coated with holey carbon film. For SEM investigations, the samples were deposited on carbon tape. The shape of the M1 needles was analyzed by measuring the length and diameter of more than 200 needles on SEM images. Based on the average length and diameter of the needles, the total surface area and the surface area of the (001) plane was calculated for an average needle for each catalyst (Table 1). These calculations were done under the assumptions that the material is $100 \%$ crystalline and each crystal is cylindrically shaped. Furthermore, the cylinder was assumed to have a circular basal plane. A SEMderived specific surface area was calculated using the surface area of the average needle and the crystallographic density of M1 (4.4 g/ $\left.\mathrm{cm}^{3}[8]\right)$.

\subsection{Nitrogen adsorption}

The specific surface areas of the catalysts were measured using an AUTOSORB-1-C physisorption/chemisorption analyzer (Quantachrome). Eleven points in the linear range of the nitrogen adsorption isotherm measured at $77 \mathrm{~K}$ have been used to calculate the BET surface area. Prior to adsorption, the samples have been degassed at $353 \mathrm{~K}$ for 2 hours.

\subsection{High-Sensitivity LEIS}

The Calipso LEIS uses a double toroidal analyzer, which combines a large acceptance angle with parallel energy analysis of the backscattered ions. This gives orders of magnitude higher sensitivity (HS-LEIS) than that of conventional LEIS equipment [20]. The required ion dose for analysis is so low that HS-LEIS analysis is essentially non-destructive ("static").

The extreme surface sensitivity of LEIS requires that organic contaminations due to transport and storage as well as carbonaceous deposits from the catalytic reactions are removed from the catalysts before analysis. This was done with an oxygen atom source (Oxford Applied Research, type MPD 21) which produces O-atoms of thermal energy. In contrast to molecular oxygen and ozone, the chemical energy of these O-atoms enables the complete removal of organic contaminations at room temperature without sputtering the surface. All samples were cleaned in this way (200 W, $5 \mathrm{~min}$ ) before analysis. Prolonged treatment had no influence on the LEIS spectra, thus confirming the cleanliness of the samples.

\subsection{Calibration of the LEIS signals}

It has been shown before that the influence of roughness on the LEIS signals is very small [21]. Thus, the precise dispersion is not of great importance for a good reference to calibrate the LEIS signals of the catalysts. High purity powder samples of $\mathrm{SiO}_{2}, \mathrm{~V}_{2} \mathrm{O}_{5}, \mathrm{Nb}_{2} \mathrm{O}_{5}, \mathrm{MoO}_{3}$ and $\mathrm{TeO}_{2}$ were, therefore, used as references to quantify the surface concentrations of $\mathrm{V}, \mathrm{Nb}, \mathrm{Mo}$ and $\mathrm{Te}$. Since the surfaces were cleaned with $\mathrm{O}$ atoms, the cations in the outer surface will be in their highest oxidation state (thus $\mathrm{TeO}_{3}$ for Te). The LEIS spectra are given in Figures 1 and 2. The lighter elements $(\mathrm{Si}, \mathrm{V})$ were quantified with $3 \mathrm{keV}$ ${ }^{4} \mathrm{He}^{+}$ions, while $5 \mathrm{keV}^{20} \mathrm{Ne}^{+}$ions were used for the heavier elements ( $\mathrm{V}, \mathrm{Nb}, \mathrm{Mo}$ and $\mathrm{Te}$ ). As can be seen in Figs. 1 and 2, the surface of the $\mathrm{SiO}_{2}$ was contaminated with a trace of iron oxide, while the $\mathrm{TeO}_{2}$ contained some cobalt- or nickel oxide (mass 59). Even with the heavier $\mathrm{Ne}$ ions, there was an overlap of the $\mathrm{Nb}$ and Mo peaks. The elements are neighbors in the periodic table and their isotopes $(\mathrm{Nb}$ : 93 ; Mo: 92-100) overlap. For the catalysts, the relative concentrations of $\mathrm{Nb}$ and Mo were determined by curve fitting. The large width of the Te peak originates from isotopic broadening (atomic masses 122 - 130). Since these masses are very different from those of the other elements, it is easy to quantify Te. V can be analyzed using both He and $\mathrm{Ne}$ ions and the two analyses give very similar results. The 


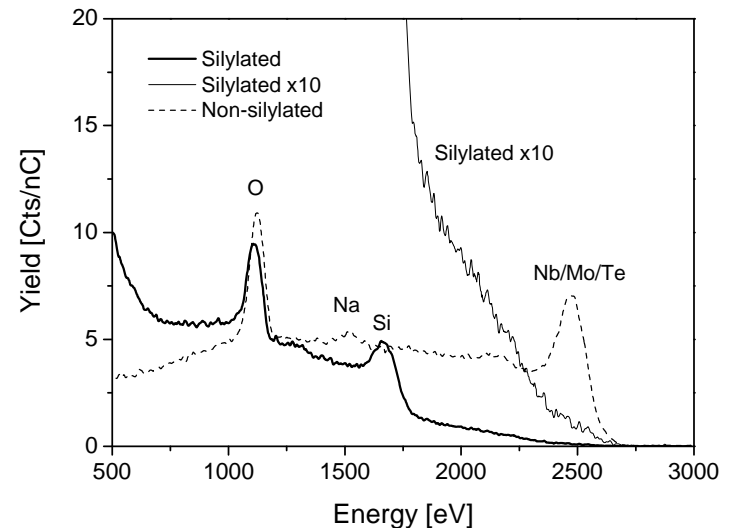

Fig. 3: $3 \mathrm{keV} \mathrm{He}{ }^{+}$scattering. Non-silylated, silylated and silylated $\mathrm{x} 10$.

presented numbers for $\mathrm{V}$ are the average of the two measurements.

The LEIS calibration gives the fractions of the surface that are composed of the various oxides. To obtain atomic concentrations, these fractions have to be corrected for the surface densities of the cations in the references. These surface densities have been estimated as $\left(\rho \cdot \mathrm{N}_{\mathrm{Av}} /\right.$ $M)^{2 / 3}$, where $\rho$ is the bulk density, $\mathrm{N}_{\mathrm{Av}}$ is Avogadro's number and $\mathrm{M}$ the molar mass. It has been shown before [22] that this estimate agrees closely with the estimate from the surface unit-cell taking the close-packed surface plane, which is dominant on powders. For $\mathrm{V}_{2} \mathrm{O}_{5}, \mathrm{Nb}_{2} \mathrm{O}_{5}, \mathrm{MoO}_{3}$ and $\mathrm{TeO}_{3}$ these densities are 9.96, 9.36, 7.28 and 7.68 (x $10^{14}$ ) metal atoms $/ \mathrm{cm}^{2}$, respectively.

\section{Results}

\subsection{Silylation of M1}

The surface of a MoVTeNbO ${ }_{x}$ mixed oxide composed mainly of the M1 phase has been covered with a layer of silica by silylation using $\mathrm{HSi}(\mathrm{OEt})_{3}$. The silylation was optimized using LEIS. Figures 3 and 4 show the LEIS spectra of the catalyst before and after silylation according to the process described in Section 2. The $3 \mathrm{keV} \mathrm{He}^{+}$spectrum before silylation shows the peaks for $\mathrm{O}, \mathrm{V}$ and a combined peak for $\mathrm{Nb}$, Mo and $\mathrm{Te}$. In addition, some $\mathrm{Na}$ contamination is observed. The $5 \mathrm{keV} \mathrm{Ne}^{+}$spectrum gives a better mass resolution for the heavier elements. It shows the peaks for $\mathrm{V}, \mathrm{Te}$ and a broad peak due to $\mathrm{Nb}$ and Mo. After silylation, only the peaks for $\mathrm{O}$ and $\mathrm{Si}$ (He spectrum) are present. No peaks are observed in the Ne spectrum. This confirms the successful complete silylation of the catalyst.

In addition to the peaks in the He spectrum there is a background extending to higher energies. This background is due to ions that were backscattered by $\mathrm{Nb}, \mathrm{Mo} / \mathrm{Te}$ in deeper layers. The most likely process being that the ions were neutralized at the first interaction with the surface,

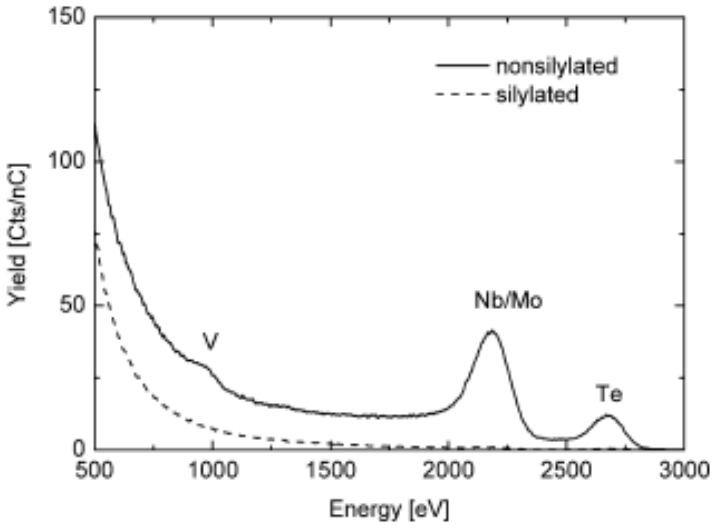

Fig. 4: $5 \mathrm{keV} \mathrm{Ne}{ }^{+}$scattering. Non-silylated and silylated.

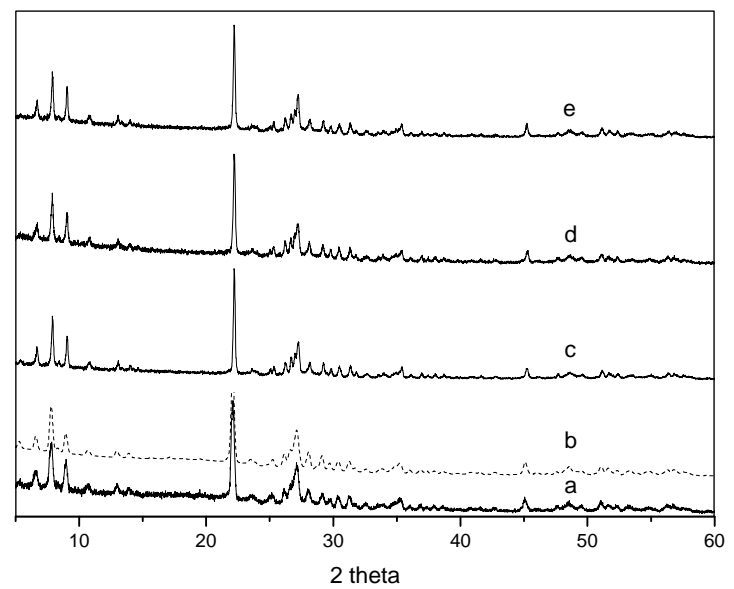

Fig. 5: XRD patterns of as synthesized M1 (a), and calculated patterns assuming phase-pure M1 [8] (b); XRD patterns of M1 after propane oxidation (c), and of silylated M1 before (d), and after (e) propane oxidation.

lost some energy along the ingoing (straggling) trajectory, backscattered in a hard binary collision with a $\mathrm{Nb}, \mathrm{Mo}$, or Te atom, and lost again some energy on the straggling way back to the surface. Since in LEIS only backscattered ions are detected, it is necessary that the backscattered He atoms are reionized in an interaction with a surface atom, just before leaving the surface. For $3 \mathrm{keV} \mathrm{He}^{+}$ions scattered by this type of oxides the straggling energy loss contributes about $160 \mathrm{eV}$ for backscattering from a depth of $1 \mathrm{~nm}$. The shape of the 10x magnified background thus gives information on the thickness distribution of the silica coating. It shows that a small fraction of the coating is only just covered (1-2 atoms thick), while the maximum thickness is about $2 \mathrm{~nm}$. Consistently, TEM (see below) shows that the silica layer is not homogeneous and its thickness varies.

\subsection{Phase composition of the MoVTeNb oxide}

With regards to the bulk structure, a material of high phase purity has been exposed to the silylating agent (Fig. 5a), which becomes evident from the comparison of the 


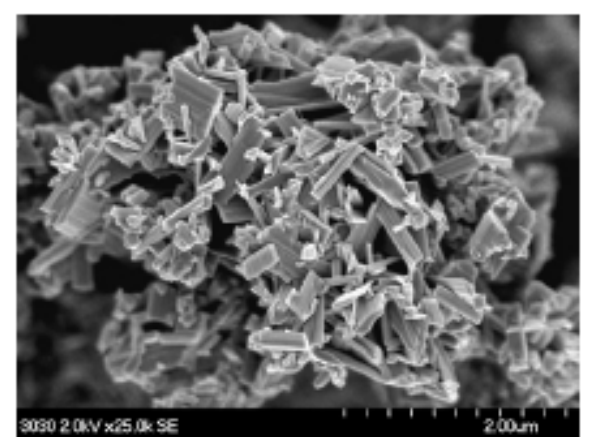

(a)

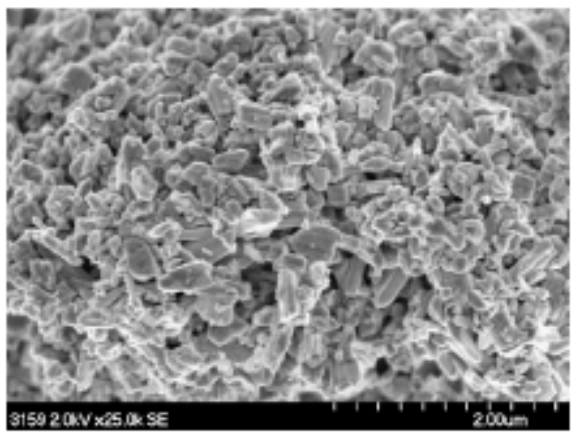

(c)

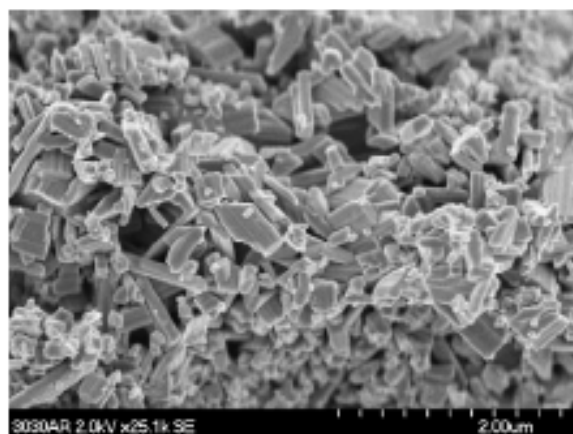

(b)

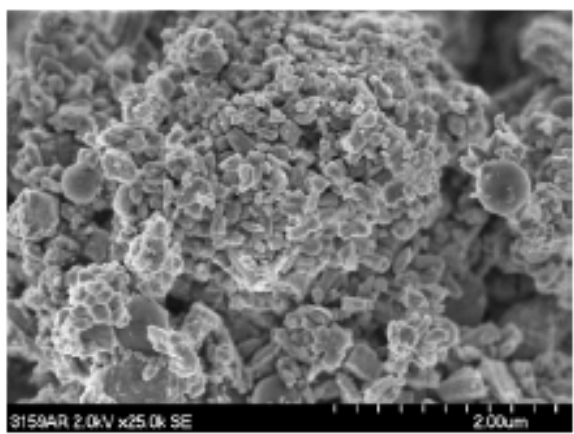

(d)

Fig. 6: SEM images of phase-pure M1 before (a) and after propane oxidation (b); SEM images of silylated M1 before (c) and after propane oxidation (d).

measured XRD patterns with the patterns calculated under the assumption that the solid is composed exclusively of M1 [8] (dotted line, Fig. 5b). Within the range of accuracy of the method, the material is phase-pure. The phase composition of the mixed oxide remained unchanged after catalytic reaction (Fig. 5c) as well as after the silylation procedure (Fig. $5 \mathrm{~d}, \mathrm{e}$ ). The materials are highly crystalline, which is also confirmed by TEM.

\subsection{Microstructure of the model catalysts}

The typical needle-shape morphology of the original phase-pure M1 material is observed in the SEM image shown in Figure 6a. Only very few particles are found that clearly differ in morphology indicating the presence of traces of other phases not detectable by XRD, or minor amounts of amorphous fractions. The needle-shape morphology is maintained after catalytic test (Fig. 6b) and after silylation (Fig. 6c). In the latter material, some spherical particles are observed that have been assigned to pure $\mathrm{SiO}_{2}$ by EDX analysis. The needle-like shape of the silylated M1 particles (Fig. 6c) is also not substantially affected by propane oxidation (Fig. 6d).

Shape analysis of the original M1 catalyst revealed a broad distribution of the needle length with a maximum between 100 and $400 \mathrm{~nm}$ (Fig. 7a). The abundance of shorter needles, especially those with length shorter than $100 \mathrm{~nm}$, clearly increases after propane oxidation (Fig. 7b), which is attributed to breakage of the needles due to the mechanical manipulation during preparation of the sieve

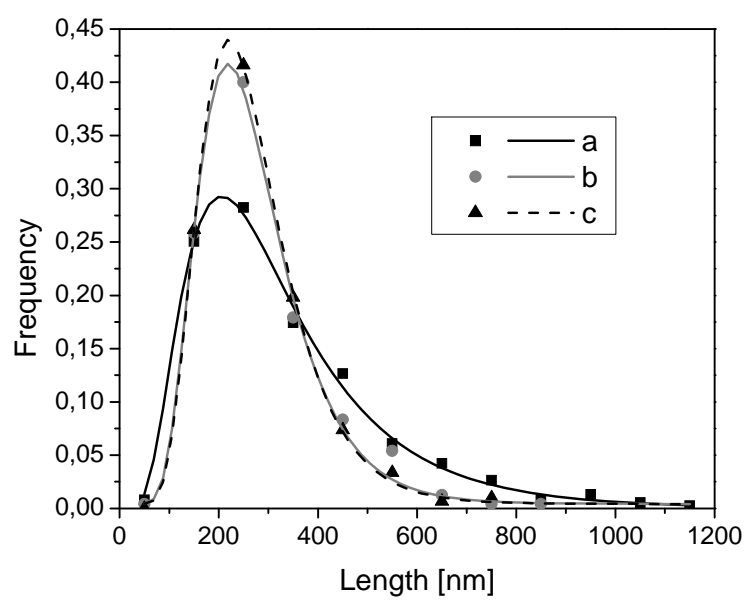

Fig. 7: Distribution of the needle length in as synthesized phasepure M1 (a), in M1 after propane oxidation (b), and in silylated M1 after propane oxidation (c). The lines on the figure are log-normal fits

fraction prior to the catalytic test. A similar length distribution has been observed with the silylated catalyst after the catalytic reaction (Fig. 7c), which indicates that the impact of mechanical treatment is comparable for silylated and non-silylated M1.

In Table 1, mean needle length, mean needle diameter and the mean diameter/length ratio of the needles obtained by shape analysis of about 300 needles are summarized for the original M1 material, the M1 catalyst after propane oxidation and the silylated M1 after the cata- 
lytic testing. As the M1 needles are naturally crystals with preferred growth direction perpendicular to the $\{001\}$ planes, a preferential breaking perpendicular to the length axis is expected during mechanical treatment. This preferential breaking is reflected in a slight increase of the mean diameter/length ratio from 0.56 for the original M1 catalyst before propane oxidation to 0.59 and 0.61 for the nonsilylated and the silylated M1, respectively, after propane oxidation. Assuming that all needles have cylindrical geometry with circular basal planes and mean geometric parameters (diameter, length) as given in Table 1, the total surface area and the surface area of the (001) planes of an average needle have been calculated for the three model catalysts (Table 1). Taking into account a unit cell density of M1 of $4.4 \mathrm{~g} / \mathrm{cm}^{3}$ [8], specific surface areas can be calculated that agree well with the BET surface areas measured (Table 1), confirming high crystallinity of the materials. If each needle was disrupted once, an increase in surface area of about $20 \%$ is expected from the cylindrical geometry. The actual increase in BET surface area of the non-silylated M1 before and after propane oxidation comprises $13 \%$, which agrees again well with the increase in specific surface area of $14 \%$ calculated based on the shape analysis confirming the reliability of the method. The BET surface area of the silylated M1 has not been measured since falsification due to the presence of silica particles was expected. However, considering the similar size distribution of nonsilylated and silylated needles after the catalytic testing (Figure 7), an increase in surface area of $13 \%$ can also be expected for the silylated catalyst.

In summary, combined shape- and BET surface area analysis clearly indicate a preferential disruption of the M1 needles perpendicular to the [001] direction by mechanical treatment prior to catalytic testing. Comparing nonsilylated and silylated M1 needles, the impact of the mechanical treatment on particle morphology is similar providing further evidence for the applicability of the present approach.

Provided that no silica is removed from the sides of the needles, the new surface area formed by mechanical treatment of completely silylated M1 is mainly composed of (001) planes (Scheme 1). Needles that exclusively expose the (001) plane, have been detected by high-resolution transmission electron microscopy in the silylated M1 catalyst after running the catalytic test reaction (Fig. 8a). The image in Fig. 8a shows one needle with the sides covered by silica and with the cross-section plane free of $\mathrm{SiO}_{2}$ confirming that the needles can break perpendicular to the length axis. Furthermore, needles completely covered by a layer of silica are also observed (Fig. 8b) attesting that not every needle is broken, which is in agreement with the results of shape analysis and nitrogen adsorption. However, partial removal of silica from the sides of the needles cannot be excluded as evidenced by the TEM image shown in Fig. 8c.
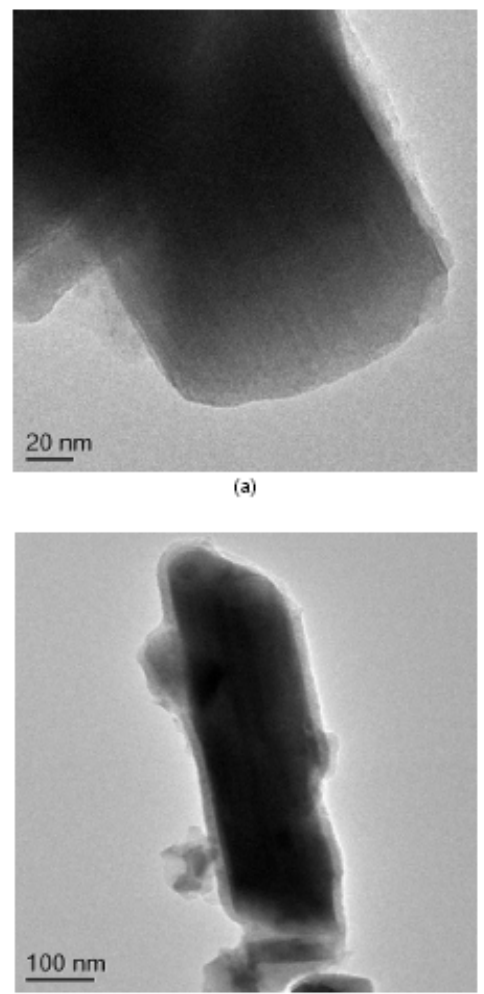

(b)

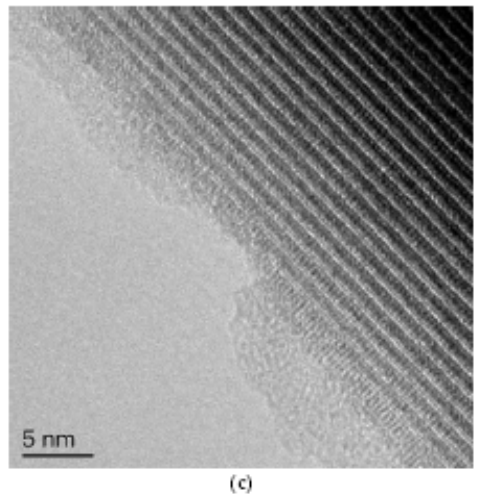

Fig. 8: HRTEM images of a silylated M1 needle disrupted along the (001) plane (a), of a completely silylated M1 needle (b) and of a silylated M1 needle with silica scratched from the sides.

\subsection{Catalytic properties of the model catalysts in pro- pane oxidation}

Pure silica does not show any catalytic activity in propane oxidation under the reaction conditions applied. Completely silylated M1 is, therefore, expected to be inactive. However, an experimental verification of this statement for a completely silylated model catalyst is impossible. The mechanical manipulation of the catalyst by pressing and sieving is required to prepare a sieve fraction prior to the catalytic test. This causes partial damage of aggregates and even of the primary particles resulting in exposure of new $\mathrm{MoVTeNbO}_{\mathrm{x}}$ surface in case of the silylated as well as in case of the non-silylated reference catalyst (Scheme 1). 
Table 2: Catalytic properties of M1 and silylated M1 in propane oxidation to acrylic acid (AA) after $32 \mathrm{~h}$ time on stream at $673 \mathrm{~K}$

\begin{tabular}{|c|c|c|c|c|c|c|c|}
\hline Catalyst & $\begin{array}{c}\mathrm{X}_{\mathrm{Cзн}} \\
(\%)\end{array}$ & $\mathrm{S}_{\mathrm{AA}}(\%)$ & $\begin{array}{l}\mathbf{Y}_{\mathrm{AA}} \\
(\%)\end{array}$ & $\begin{array}{c}\mathrm{C}_{3} \mathrm{H}_{8} \text { con- } \\
\text { sumption rate } \\
{\left[\text { mmol }_{\mathrm{C} 3 \mathrm{H} 8} /\right.} \\
\left.\text { h g gat }_{\text {cat }}\right]\end{array}$ & $\begin{array}{c}\mathrm{C}_{3} \mathrm{H}_{8} \text { consump- } \\
\text { tion rate[mmol } \\
\mathrm{CuH}^{2} / \\
\mathbf{h ~ m}_{\text {exp.surf. }}^{2}\end{array}$ & $\begin{array}{c}\text { formation rate } \\
\text { AA } \\
{\left[\mathrm{mmol}_{\mathrm{AA}} / \mathbf{h} \cdot \mathrm{g}_{\mathrm{cat}}\right]}\end{array}$ & $\begin{array}{c}\text { formation rate AA } \\
{\left[\mathbf{m m o l}_{\mathrm{AA}}\right.} \\
\left./ \mathbf{h} \cdot \mathbf{m}^{2} \text { exp.surf. }\right]\end{array}$ \\
\hline M1 & 49 & 73 & 36 & $>2.94$ & $>0.39$ & $>2.15$ & $>0.28$ \\
\hline $\begin{array}{c}\text { Silylated } \\
\text { M1 }\end{array}$ & 9 & 100 & 9 & 0.54 & 0.24 & 0.54 & 0.24 \\
\hline
\end{tabular}

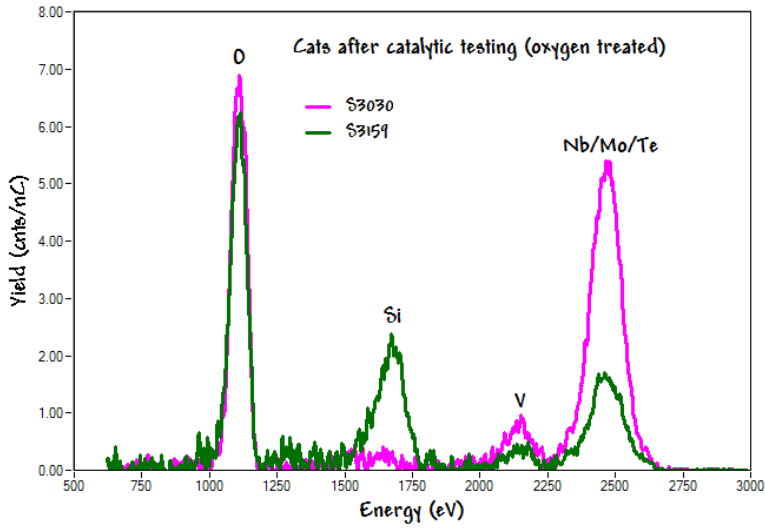

Fig. 9: Background-subtracted $3 \mathrm{keV}{ }^{4} \mathrm{He}^{+}$spectra from catalytically tested samples.

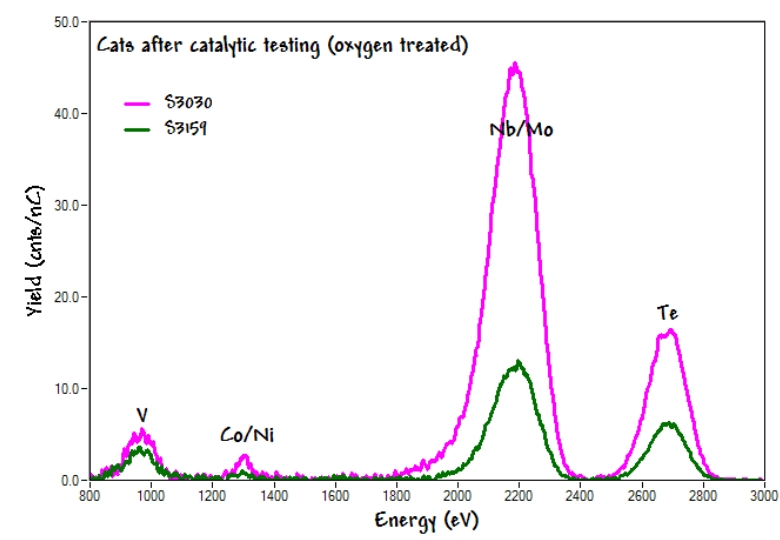

Fig.10: $5 \mathrm{keV}^{20} \mathrm{Ne}^{+}$spectra from catalytically tested samples.

The catalytic performance of the two mechanically treated catalysts is compared in Table 2. Propane oxidation was performed in parallel reactors applying the same temperature, space velocity and feed composition. Under steady-state conditions, the conversion of propane over the reference M1 is by a factor of five higher than over the silylated model catalyst. Whereas only acrylic acid is formed over silylated M1, the selectivity is lower for the reference material producing $12 \% \mathrm{CO}_{2}$ and $15 \% \mathrm{CO}$ as byproducts. These differences in selectivity are most probably related to the different propane conversion levels. The carbon oxides might be formed in consecutive reactions, thus lowering the selectivity to acrylic acid over the refer- ence M1. In Table 2, average rates are shown. Due to the high conversion of the non-silylated reference catalyst, the average consumption rate of propane is underestimated for this catalyst. Nevertheless, it is five times higher compared to the silylated catalyst. The higher $\mathrm{MoVTeNbO}_{\mathrm{x}}$ surface area that is exposed to the reactants in case of the nonsilylated reference catalyst might be an explanation for this observation. The surface area of the reference M1 has been measured by nitrogen adsorption (Table 1). For determination of the accessible $\mathrm{MoVTeNbO}_{x}$ surface area of the silylated catalyst, LEIS has been applied. The procedure of the LEIS measurement is described in the following section.

\subsection{LEIS after catalysis}

To obtain a proper comparison between the catalysts, the silylated and non-silylated samples were both investigated with LEIS after the same mechanical treatment (pelletizing, crushing, sieving) and catalytic testing (see Scheme 1). The He and Ne spectra, after background subtraction, are given in Figs. 9 and 10. Due to partial disruption of the needles, significant signals are now observed for $\mathrm{V}, \mathrm{Nb}, \mathrm{Mo}$, and $\mathrm{Te}$ in the silylated sample. Again, some $\mathrm{Co} / \mathrm{Ni}$ contamination is present. Using the reference spectra (Figs. 1 and 2) the surface fraction of the elements in Figs. 9 and 10 can be quantified. It is found that the sum of the surface fractions of $\mathrm{V}_{2} \mathrm{O}_{5}, \mathrm{Nb}_{2} \mathrm{O}_{5}, \mathrm{MoO}_{3}$ and $\mathrm{TeO}_{3}$ in the treated silylated sample amounts to $30 \%$ of that in treated non-silylated sample. The other part of M1 (70\%) remains covered by $\mathrm{SiO}_{2}$. The $30 \%$ are thus the new surface that is generated by the mechanical treatment.

To obtain the atomic surface concentrations, the surface fractions of the oxides have to be corrected for the different numbers of metal atoms $/ \mathrm{cm}^{2}$ in these oxides. In Table 3, the atomic compositions (in brackets normalized to Mo) are given for the treated non-silylated sample and the new surface of the silylated sample. The results of the bulk analyses by EDX are given for comparison. In agreement with earlier studies [15], the chemical composition of the outermost surface layer differs significantly from that of the bulk. The LEIS measurement of the M1 reference provides integral information including the basal plane and the 
Table 3: Metal composition of the bulk (EDX) and topmost layer (LEIS) in at-\%. The numbers in parenthesis represent molar ratios of the metals normalized to molybdenum

\begin{tabular}{|c|c|c|c|c|c|}
\hline Catalyst & Method & Mo & $\mathbf{V}$ & Te & $\mathrm{Nb}$ \\
\hline \multirow{2}{*}{$\mathbf{M 1}^{1}$} & EDX & $64(1)$ & $22(0.34)$ & $\begin{array}{ll}5 & (0.08) \\
\end{array}$ & $9 \quad(0.14)$ \\
\hline & LEIS & n.d. & n.d. & n.d. & n.d. \\
\hline \multirow[t]{2}{*}{$\mathbf{M 1}^{2}$} & EDX & $64(1)$ & $20(0.31)$ & $6 \quad(0.09)$ & $10(0.16)$ \\
\hline & LEIS & $65(1)$ & $12(0.18)$ & $12(0.18)$ & $11(0.17)$ \\
\hline \multirow{2}{*}{ silylated $\mathbf{M 1}^{1}$} & EDX & $63(1)$ & $22(0.35)$ & $6(0.09)$ & $\begin{array}{ll}9 & (0.14) \\
\end{array}$ \\
\hline & LEIS & 0 & 0 & 0 & 0 \\
\hline \multirow[t]{2}{*}{ silylated $\mathrm{M1}^{2}$} & EDX & $62(1)$ & $21(0.34)$ & $\begin{array}{ll}6 & (0.09) \\
\end{array}$ & $11(0.18)$ \\
\hline & LEIS & $55(1)$ & $20(0.37)$ & $15(0.27)$ & $10(0.17)$ \\
\hline
\end{tabular}

1 as synthesized

2 catalyst after propane oxidation

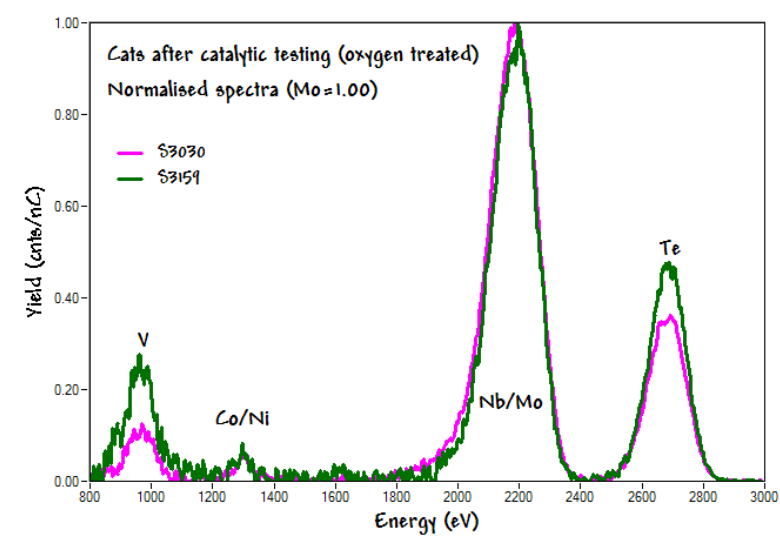

Fig. 11: Normalised $5 \mathrm{keV}^{20} \mathrm{Ne}^{+}$spectra $(\mathrm{Mo}=1.00)$ from catalytically tested sample (after subtraction of background).

lateral surface showing an enrichment of tellurium at the expense of vanadium. The situation on the freshly formed surface of the silylated model catalyst, which is dominated by the (001) plane, is different. Here, an increase of tellurium on account of molybdenum was observed. No depth profile and no differences between the catalysts are noticed with respect to the $\mathrm{Nb}$ concentration. The clear differences in the surface compositions are also illustrated in Fig. 11, where the Ne spectra have been normalized on the maximum of the $\mathrm{Nb} / \mathrm{Mo}$ peak.

\section{Discussion}

It has been well documented that the M1 phase occurring in MoVTeNb mixed oxide catalysts possesses a key function in selective oxidation and ammoxidation of propane $[6,14,23]$. The crystal structure hosts four different metals, three of them with variable oxidation state, providing the requirements to catalyze redox-reactions by exposing or bearing structural arrangements at the surface that cope in a distinguished way with the demands of propane activation and the transfer of 8 electrons in its selective oxidation to acrylic acid or in its ammoxidation to acrylonitrile, respectively. Surface and sub-surface reduction and reoxidation of M1 crystals seems to be possible without structural collapse [24]. The occupancy of the 13 metal sites in the unit cell of the crystal structure is variable [9$10,14]$. Accordingly, the catalytic properties may be controlled by synthesis or post-treatment procedures. Crystallinity seems to be required to obtain catalytic activity for selective (amm)oxidation of propane [6, 25-26]. The possible role of defects remains an open question [24, 27]. Typically, nano-crystalline M1 composed of fine needles of a few hundred nanometers in length exhibits excellent catalytic behavior.

Phase-pure M1 synthesized in the present study shows the corresponding morphology and possesses very small amounts of impurity phases or amorphous fractions as evidenced by XRD (Fig. 5) and electron microscopy (Fig. 6a). Compared to published M1 stoichiometries, e.g., $\mathrm{Mo}_{1} \mathrm{~V}_{0.15} \mathrm{Te}_{0.12} \mathrm{Nb}_{0.13} \mathrm{O}_{\mathrm{x}}$ [8] or $\mathrm{Mo}_{1} \mathrm{~V}_{0.23} \mathrm{Te}_{0.11} \mathrm{Nb}_{0.14} \mathrm{O}_{\mathrm{x}}$ [9], the present material is characterized by an increased $\mathrm{V}$ content and a reduced Te content (Table 3), which might be the reason for its exceptional catalytic behavior (Table 2). The average formation rate of acrylic acid measured in the stationary state after 32 hours on stream is $2.15 \mathrm{mmol} / \mathrm{h}$ $\mathrm{g}_{\text {cat }}$, which exceeds results reported for M1 in the literature [28]. Due to the phase purity and high catalytic activity of the present material, it has been chosen in the present attempt to prepare a model catalyst preferentially exposing the (001) plane. Based on grinding experiments, and under the assumption that the structural characteristics and the chemical composition of the surface do not differ from the bulk even under reaction conditions, it was previously concluded that the catalytically active sites in propane oxidation $[11,12]$ or ammoxidation $[14,26]$ are located on terminating (001) planes. This assumption is at least not consistent with room temperature LEIS measurements that give an indication of gradients in elemental composition between surface and bulk of M1 [29]. Surface texturing of catalyst particles has also been revealed by high-resolution transmission electron microscopy on the surface of high performing $\mathrm{MoVTeNbO}_{\mathrm{x}}$ catalysts that have been leached with solvents [27]. In any case, a well-defined and rigid arrangement of structural units might be rather unlikely at reaction temperature and in the presence of steam in the feed. Addressing the question by in-situ spectroscopic methods is, however, quite challenging. Therefore, in the present study we have used propane oxidation itself as a probe reaction to ascertain the specific catalytic activity of 
the (001) plane compared to the integral activity of the entire M1 surface. For these purposes, the M1 crystals have been covered completely by a thin layer of silica. HS-LEIS confirms coverage of the whole mixed oxide surface (Fig. 4). The thickness of the layer ranges from a few monolayers to $20 \mathrm{~nm}$ as verified by LEIS and TEM. Neither the particle size distribution of the original M1 (Table 1) nor its chemical composition (Table 3 ) is affected by the silylation procedure. Before introduction into the catalytic test reactor, the powders have been pelletized, crushed and sieved. Such a gentle mechanical treatment leads to partial disruption of needles and formation of fresh MoVTeNbOx surface. In case of the silylated M1, the newly emerging MoVTeNb oxide surface has been veryfied by HS-LEIS (Fig. 4). For the pure, non-silylated M1, these morphological changes should be reflected in an increased specific surface area measured by nitrogen adsorption. Under the assumption that the mechanical treatment leads exclusively to a breakage of needles perpendicular to the [001] direction, the difference corresponds to the surface of newly formed (001) planes. In the present experiment, the difference in the specific surface area is $13.4 \%$ (Table 1). A quite similar result $(14.3 \%)$ is obtained if the mean particle size determined based on the shape analysis by SEM and the bulk density of M1 $\left(4.4 \mathrm{~g} / \mathrm{cm}^{3}\right.$ [8]) are used to calculate the increase in surface area of pure, non-silylated M1 (Table 1), which confirms satisfactorily the reliability of the shape analysis and which shows that the mechanical treatment applied is mainly associated with breakage of needles and not with other damage. The relevance of shape analysis is an important fact, since in case of the silylated catalyst we cannot rely on BET surface area measurements for calculation due to an expected increase in surface area caused by the possibly not smooth $\mathrm{SiO}_{2}$ layer and the occasional formation of pure silica particles.

The breaking probability of the original M1 needles as well as the silylated needles is low (Table 1, Fig. 7) and long needles are more frequently broken than short needles (Fig. 7). Figure 7 confirms that non-silylated and silylated needles break in a similar way, since the particle size distribution of the two materials after the catalytic test is essentially the same. For the silylated catalyst, the increase in surface area, i.e., the yield in newly formed basal plane surface area corresponds to $12.7 \%$ when the mean particle size determined by shape analysis is used for the calculation (Table 1).

In summary, based on shape analysis and/or nitrogen adsorption before and after catalytic test, the breakage of the M1 needles benefits in newly formed (001) surface area of approximately $15 \%$ both for original and silylated M1.

As an independent method, LEIS analysis has been applied to determine the freshly formed MoVTeNbOx surface of the silylated catalyst. This fraction corresponds to $30 \%$ of the surface of the original M1. This value is twice as high as the increase calculated from shape analysis implying that not only silica-free (001) planes have been generated. HRTEM (Fig. 8c) shows that some silica has been scratched from the sides of the needles as well. Assuming that the increase in surface area of about $15 \%$ calculated from shape analysis (Table 1) corresponds exclusively to the newly formed silica-free (001) planes, the ratio of the basal plane area versus other $\mathrm{MoVTeNbO}_{\mathrm{x}}$ surface area, i.e., lateral surface area, is about 1 in the silylated M1. In the original M1, the entire surface of the needles is exposed to the gas phase during propane oxidation and, therefore, the ratio of (001) surface area to lateral surface area corresponds to 0.25 (Table 1). Consequently, albeit other surface planes are to some extent also exposed to the reactants in the silylated M1 during propane oxidation, we can conclude that the silylated, mechanically treated M1 catalyst represents a suitable model system for studying the catalytic properties of the (001) plane, since half of the exposed $\mathrm{MoVTeNbO}_{\mathrm{x}}$ surface is composed of (001) planes.

The catalytic performance of M1 and silylated M1 is compared in Table 2. As expected, the conversion of silylated M1 is much lower than that of M1, since less MoV$\mathrm{TeNbO}_{\mathrm{x}}$ surface is accessible in the former catalyst. Based on the information obtained from the HS-LEIS study that actually $30 \%$ new $\mathrm{MoVTeNbO}_{\mathrm{x}}$ surface is available in the silylated catalyst, consumption rates of propane normalized to the area of exposed $\mathrm{MoVTeNbO}_{\mathrm{x}}$ surface have been calculated considering $7.6 \mathrm{~m}^{2} / \mathrm{g} \mathrm{MoVTeNbO}_{\mathrm{x}}$ surface for M1 (BET surface area given in Table 1) and $2.3 \mathrm{~m}^{2} / \mathrm{g}$ $\mathrm{MoVTeNbO}_{\mathrm{x}}$ surface for silylated M1 $\left(0.30 \times 7.6 \mathrm{~m}^{2} / \mathrm{g}\right)$. The rates are quite similar for both catalysts. That means that the intrinsic catalytic property of the entire M1 surface is similar to the catalytic behavior of a surface that is composed by $50 \%$ of $(001)$ planes. This finding makes the uniqueness of the basal plane of M1 at least doubtful.

The result is especially surprising since the chemical composition of the newly formed M1 surface of the silylated catalyst, which can at least partially be regarded as the inner surface of M1, is of different chemical composition than the average surface composition of M1 as shown by the LEIS experiment. In Table 3 the bulk composition measured by EDX and the surface composition measured by HS-LEIS are compared. Whereas the EDX bulk compositions of the samples are almost the same confirming that the silylation procedure does not affect the bulk composition, the HS-LEIS surface composition of the newlygenerated surface shows relatively large differences, specifically, an increased concentration of tellurium at the expense of molybdenum. However, prior to the present LEIS measurement, a mild pre-treatment with oxygen atoms at room temperature was necessary to remove environmental contaminations from reaction, transport, and storage. This procedure, which converts all the surface metal ions into their highest oxidation states, may be connected with structural changes. Although the local structure will be affected, it is not sure whether the elemental composition will change much. Moreover, the surface reconstruction holds for the entire surface of M1 thus justifying the comparison of the basal plane with the total surface in terms of elemental composition.

In any case, these uncertainties do not affect the findings that the intrinsic catalytic properties of the basal plane 
of $\mathrm{M} 1$ in the selective oxidation of propane to acrylic acid do not differ much from those of the lateral surface of the M1 needles. Accepting this result, the question arises why increased catalytic activity and improved selectivity to acrylic acid have been observed in previous studies after grinding of M1 [11, 12]. The details of the grinding procedure were not given in the corresponding publications. However, most likely, the mechanical treatment was less gentle than in the present case, probably, generating a more defect-rich material in addition to the formation of extra basal planes. Another explanation could be that contaminations, such as residues from the preparation, which are common on the surface of catalysts, were removed by extended grinding. Breaking the needles and scratching deposited impurities away from the sides of the needles would expose fresh, uncontaminated, thus active MoV$\mathrm{TeNb}$ oxide surface.

\section{Conclusions}

The model studies presented here provide an indication that a distinguished lattice plane of the M1 crystal structure, the (001) plane, is not solely responsible for its outstanding catalytic activity and selectivity in the partial oxidation of propane to acrylic acid. The chemical and structural nature of the active ensembles on the catalyst surface remains unknown. The unique crystal structure of the M1 phase certainly plays an essential role in the selec-

\section{References}

[1] T. Ushikubo, H. Nakamura, Y. Koyasu, S. Wajiki, US Patent 5380933 (1995) to Mitsubishi Kasei Corporation.

[2] M. Hatano, A. Kayo, US Patent 5049692 (1991) to Mitsubishi Kasei Corporation.

[3] T. Ushikubo, K. Oshima, A. Kayou, M. Hatano, Stud. Surf. Sci. Catal. 112 (1997) 473.

[4] M. Baca, A. Pigamo, J.L. Dubois, and J.M.M. Millet, Topics in Catalysis 23 (2003) 39.

[5] J.M. Oliver, J.M. Lopez Nieto, P. Botella, Catalysis Today 96 (2004) 241.

[6] W.Ueda, D.Vitry, T. Katou, Catalysis Today 96 (2004) 235.

[7] H. Tsuji, K. Oshima, Y. Koyasu, Chem. Mater. 15 (2003) 2112.

[8] P. DeSanto Jr., D.J. Buttrey, R.K. Grasselli, C.G. Lugmair, A.F. Volpe Jr., B.H. Toby, T. Vogt, Z. Kristallogr. 219 (2004) 152

[9] H. Murayama, D. Vitry, W. Ueda, G. Fuchs, M. Anne, J.L. Dubois, Applied Catalysis A: General 318 (2007) 137.

[10] A. Celaya Sanfiz, T.W. Hansen, F. Girgsdies, O. Timpe, E. Rödel, T. Ressler, A. Trunschke, R. Schlögl, Topics in Catalysis, accepted.

[11] W. Ueda, K. Oshihara, Appl. Catal. A 200 (2000) 135

[12] K. Oshihara, T. Hisano, W. Ueda, Topics in Catalysis 15 (2001) 153.

[13] V.V. Guliants, R. Bhandari, R.S. Soman, O. Guerrero-Perez, M.A. Banares, Appl. Catal. A 274 (2004) 213. tive (amm)oxidation of propane, since amorphous material or MoVTeNb oxide composed of different phases cannot compare to phase-pure, crystalline M1. All experimental experience evidences that this might be different from related mixed oxides, such as nano-structured MoVW oxides, which have been reported to be active in propylene oxidation in a semi-crystalline state [30]. Similar intrinsic reactivity irrespective of the terminating lattice plane implies that related active ensembles of metal-oxo clusters are exposed to the reactants on the entire surface of the M1 needles. The lateral surface of the needles accounts for the main part of the surface area of M1 $(80 \%)$. The stepped morphology of the latter surface may generate similar metal-oxo arrangements like on the surface of the basal plane of the needles. HR-TEM studies together with targeted synthesis of M1 showing different microstructure are currently in progress to verify this assumption.

\section{Acknowledgement}

The authors thank Dr. Olaf Timpe for helpful discussions, Dr. Frank Girgsdies for performing phase analysis of the catalysts, Edith Kitzelmann for conducting the XRD measurements, Kilian Klaeden, and Gisela Lorenz for carrying out the nitrogen adsorption measurements.

[14] R.K. Grasselli, D.J. Buttrey, P. DeSanto, Jr., J.D. Burrington, C.G. Lugmair, A.F. Volpe, Jr., T. Weingand, Catalysis Today 91-92 (2004) 251.

[15] [15] V.V. Guliants, R. Bhandari, B. Swaminathan, V.K. Vasudevan, H.H. Brongersma, A. Knoester, A.M. Gaffney and S. Han, J. Phys. Chem. B 109 (2005) 24046

[16] V.V. Guliants, R. Bhandari, A.R. Hughett, S. Bhatt, B.D. Schuler, H.H. Brongersma, A. Knoester, A.M. Gaffney and S. Hann, J. Phys. Chem. B 110 (2006) 6129.

[17] J.-P. Jacobs, A. Maltha, J.G.H. Reintjes, J. Drimal, V. Ponec and H.H. Brongersma, J. Catal. 147 (1994) 294.

[18] H.H. Brongersma, M. Draxler, M. de Ridder and P. Bauer, Surface Science Reports 62 (2007) 63.

[19] G. Mestl, N.F.D. Verbruggen, H. Knözinger, Langmuir 11 (1995) 3034.

[20] F.G.J.A. Hellings, H. Ottevanger, S.W. Boelens, C.L.C.M. Knibbeler, H.H. Brongersma, Surf. Sci. 162 (1985) 913.

[21] W.P.A. Jansen, A. Knoester, A.J.H. Maas, P. Schmitt, A. Kytökivi, A.W. Denier van der Gon, H.H. Brongersma, Surf. Interface Anal.36 (2004) 1469.

[22] L.C.A. van den Oetelaar, H.E. van Benthem, J.H.J.M. Helwegen, P.J.A. Stapel, H.H. Brongersma, Surf. Interf. Anal. 26 (1998) 537.

[23] M. Baca, A. Pigamo, J.L. Dubois, and J.M.M. Millet, Topics in Catalysis 23 (2003) 39.

[24] R.K. Grasselli, D.J. Buttrey, J.D. Burrington, A. Andersson, J. Holmberg, W. Ueda, J. Kubo, C.G. Lugmair, A.F. Volpe Jr, Topics in Catalysis 38 (2006) 7. 
[25] R.K. Grasselli, Catalysis Today 99 (2005) 23.

[26] R.K. Grasselli, J.D. Burrington, D.J. Buttrey, P. De Santo, C.G. Lugmair, A.F. Volpe Jr., T. Weingand, Topics in Catalysis 23 (2003) 5.

[27] .B. Wagner, O. Timpe, F.A. Hamid, A. Trunschke, U. Wild, D.S. Su, R.K. Widi, S.B.A. Hamid, R. Schlögl, Topics in Catalysis 38 (2006) 51.
[28] D. Vitry, Y. Moriwaka, J. L. Dubois and W. Ueda, Applied Catalysis A: General 251 (2003) 411.

[29] V.V. Guliants, R. Bhandari, H.H. Brongersma, A. Knoester, A.M. Gaffney, S. Han, J. Phys. Chem. B 109 (2005) 10234.

[30] H. Hibst, F. Rosowski, G. Cox, Catalysis Today 117 (2006) 234. 